\title{
Extracorporeal high-frequency combined with contrast-enhanced ultrasound: a novel imaging method for detection and treatment evaluation of patients with cervical trachea-associated relapsing polychondritis
}

\author{
Jiaxing Tang ${ }^{1}$, Weihua $\mathrm{He}^{2}$, Yuxin Zhang ${ }^{2}$, Qing Tang ${ }^{2}$, Niantu $\mathrm{He}^{2}$, Zechun Liang ${ }^{2}$, Shiyue $\mathrm{Li}^{1}$ \\ ${ }^{1}$ National Clinical Research Center for Respiratory Disease, State Key Laboratory of Respiratory Disease, Guangzhou Institute of Respiratory \\ Health, The First Affiliated Hospital of Guangzhou Medical University, Guangzhou, China; ${ }^{2}$ Department of Ultrasound, The First Affiliated \\ Hospital of Guangzhou Medical University, Guangzhou, China \\ Contributions: (I) Conception and design: J Tang, Q Tang, S Li, W He; (II) Administrative support: N He, Y Zhang; (III) Provision of study materials \\ or patients: W He; (IV) Collection and assembly of data: Z Liang; (V) Data analysis and interpretation: Y Zhang; (VI) Manuscript writing: All \\ authors; (VII) Final approval of manuscript: All authors. \\ Correspondence to: Qing Tang. Department of Ultrasound, The First Affiliated Hospital of Guangzhou Medical University, No. 151 Yanjaing Road, \\ Yuexiu Dist., Guangzhou 510120, China. Email: py_tangqing@163.com; Shiyue Li. National Clinical Research Center for Respiratory Disease, State \\ Key Laboratory of Respiratory Disease, Guangzhou Institute of Respiratory Health, The First Affiliated Hospital of Guangzhou Medical University, \\ Guangzhou 510120, China. Email: lishiyue@188.com.
}

Background: This study aims to evaluate the clinical utility of extracorporeal high-frequency ultrasound (EHFU) combined with contrast-enhanced ultrasound (CEUS) for the diagnosis and treatment of cervical trachea-associated relapsing polychondritis (CT-RP).

Methods: From January 2013 to January 2020, 24 patients with CT-RP diagnosed clinically and pathologically were retrospectively reviewed. We used EHFU to measure the thickness of trachea cartilage and the minimum internal transverse diameter of trachea and compared it with CT. The EHFU and CEUS imaging features were compared before and after treatment and used to evaluate the effects of therapy.

Results: EHFU revealed the entire cervical tracheal cartilage (136 rings, of which 124 were abnormal). The average thickness of the 124 tracheal cartilage rings was $3.657 \pm 0.52 \mathrm{~mm}$ on EHFU and $3.32 \pm 0.76 \mathrm{~mm}$ on CT $(t=1.482, \mathrm{P}>0.05)$, while the diameter of the tracheal segment was $11.98 \pm 3.22 \mathrm{~mm}$ on EHFU and $10.8 \pm 2.92 \mathrm{~mm}$ on CT $(t=1.005, \mathrm{P}>0.05)$. After treatment, most patients $(75 \%)$ showed no recurrence, and ultrasound revealed that the tracheal ring thickness was restored and the transverse diameter of the tracheal cavity was widened. Differences in EHFU measurements before and after treatment were statistically significant (both $\mathrm{P}<0.01$ ). CEUS revealed that the tracheal cartilage layer was damaged in six cases $(25 \%)$ and included structural destruction, deformation, and thinning. Follow-up evaluation revealed that the treatment outcomes of these cases were poor. The mean ultrasound examination time per patient was $10.0 \pm 2.3 \mathrm{~min}$.

Conclusions: EHFU combined with CEUS clearly and quickly revealed the CT-RP cervical tracheal wall, diameter of the trachea, and microstructural changes in tracheal cartilage. The effects of treatment can be reliably assessed by measuring reductions in tracheal cartilage thickening and echo changes before and after treatment. Dynamic monitoring of the condition provides timely and detailed information on cervical tracheal wall lesions and is valuable for clinical evaluation.

Keywords: Relapsing polychondritis (RP); extracorporeal high-frequency ultrasound (EHFU); contrast-enhanced ultrasound (CEUS); computed tomography (CT); damaged tracheal cartilage

Submitted Nov 02, 2021. Accepted for publication Dec 17, 2021.

doi: $10.21037 /$ atm-21-6175

View this article at: https://dx.doi.org/10.21037/atm-21-6175 


\section{Introduction}

Relapsing polychondritis (RP) is an uncommon acquired autoimmune inflammatory disease clinically characterized by recurrent destruction of cartilage tissue (1). The RP was occurred most frequently at the ear, nose, throat, trachea, eyes, joints, heart valves, blood vessels, and other organs, and about half of them involve the larynx, trachea and bronchus. The disease recurs readily and may exhibit an acute onset or sudden exacerbation, but can be alleviated. Airway involvement is one of the most serious complications $(2,3)$, and damage to tracheal cartilage followed by collapse of the tracheobronchial system can cause tracheal stenosis, triggering a series of dyspnea symptoms or even death (4). Early detection and assessment of cervical tracheal involvement is essential for timely and effective treatment, especially during acute inflammation $(3,5,6)$.

Currently, airway computed tomography (CT) and tracheoscopy are the most used diagnostic techniques (2). Although CT can reveal the anatomy of the airway wall and tracheal cavity, its ability to detect more subtle tracheal lesions is limited. In addition, CT is radioactive, expensive and cannot be performed at bedside $(7,8)$. It has been reported that endobronchial ultrasonography (EBUS) can reveal changes in the tracheobronchial cartilage (9). EBUS not only the surface and lumen of trachea, but also the structure of bronchial wall can be observed. Early detection of changes in trachea and bronchial cartilage is helpful for diagnosis. However, EBUS is invasive and intracavitary, with cumbersome operation and poor patient compliance. With recent developments in ultrasound technology, extracorporeal high-frequency ultrasound (EHFU) has been used to detect cervical tracheal diseases. EHFU exhibits good acoustic permeability and high resolution (10), is not associated with radiation, and is convenient. Several reports on the use of EHFU to detect cervical tracheal diseases have appeared (11-13).

In trachea-associated RP (CT-RP) patients, the mucosal tissues of the airway wall are damaged and thickened because of repeated attacks of bronchitis (2). The structures of the various layers of the pathological tracheal wall are not very clear on grayscale ultrasonography, because their echoes are similar, and are also unclear on CT. As contrastenhanced ultrasound (CEUS) provides microcirculation perfusion images of vascular cisternae (14) and can be expected to identify tracheal cartilage lesions more clearly than grayscale ultrasonography, we explored whether
EHFU combined with CEUS could serve as an effective diagnostic tool for convenient CT-RP detection and evaluation of therapies. We present the following article in accordance with the STROBE reporting checklist (available at https://dx.doi.org/10.21037/atm-21-6175).

\section{Methods}

\section{Study subjects}

We retrospectively analyzed a consecutive case series of patients with CT-RP confirmed clinically and pathologically via tracheoscopy and airway CT, from January 2013 to January 2020 at the Guangzhou Institute of Respiratory Disease. All procedures performed in this study involving human participants were in accordance with the Declaration of Helsinki (as revised in 2013). This study was approved by the Scientific Research Ethics Review Committee of The First Affiliated Hospital of Guangzhou Medical University [No. (2021)140], and all subjects gave written informed consent. Patients included in the study met all the following three criteria: (I) a CT-RP diagnosis; (II) performance of cervical CT and EHFU; (III) a follow-up time $\geq 6$ months. The exclusion criteria were as follows: (I) the cervical tracheal ring was not damaged; and (II) those with incomplete clinicopathological data.

\section{Research methods}

The instruments used were as follows: IU22 (probe frequency, 5-12 MHz; Philips, The Netherlands) and M9 (probe frequency, 5-12 MHz; Mindray, China) ultrasonography machines, a Somato Definition AS 128 CT scanner (Siemens, Germany), and a B260F CT bronchoscope (Olympus, Japan).

For EHFU scanning, each patient was placed supine with pads under the shoulders, and the head and neck extended to fully expose the neck. A high-frequency probe was used to continuously scan from the annular tracheal cartilage to the upper edge of the sternal notch (in both crosssectional and sagittal modes). We observed the structure and morphology of the tracheal rings and determined the thickness of the tracheal cartilage and minimum internal transverse diameter of the trachea. When measuring the tracheal cartilage, an abnormal echo from the cartilage ring was detected, and the thickness of the anterior midline tracheal cartilage in the tracheal cross-section was measured at this point. The images and measurements were then 

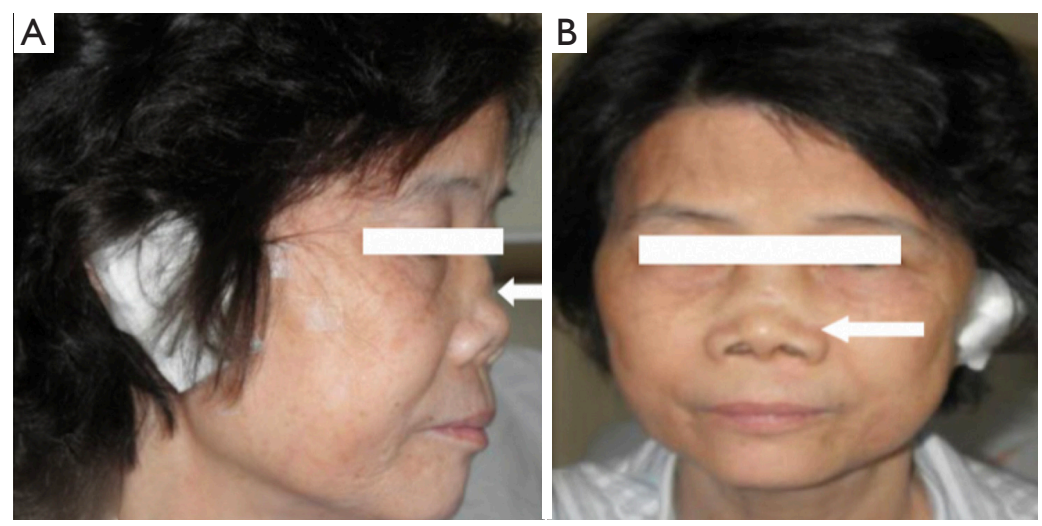

Figure 1 Case 1: a CT-RP patient (female, 66 years of age) with a typical saddle-nose bridge (arrows). (A) Lateral view; (B) top view. These images are published with the patient/participant's consent. CT-RP, cervical trachea-associated relapsing polychondritis.

saved. When measuring the intratracheal diameter, airmucosa interfaces were drawn on cross-sections and the smallest value was recorded, i.e., the minimum internal transverse diameter of the trachea.

For CEUS scanning, a sulfur hexafluoride microbubble freeze-dried powder $(59 \mathrm{mg})$ was suspended in normal saline $(5 \mathrm{~mL}, 8 \mu \mathrm{g} / \mathrm{mL})$. The contrast agent for CEUS was shaken for $30 \mathrm{~s}$, and $4.8 \mathrm{~mL}$ was immediately injected into the median cubital vein followed by $5 \mathrm{~mL}$ normal saline (to flush the tube). The scan site was the same as for EHFU.

For airway CT, the cervical tracheal cartilage ring corresponding to that evaluated by ultrasound was identified, and the thickness of the anterior midline tracheal cartilage and minimum internal transverse diameter of the trachea were measured. The images and measurements were then saved. All tests were repeated three times and the measurements were averaged.

All patients received respiratory interventions, hormonal therapy, symptomatic and supportive treatments, and other conventional treatments (depending on their needs) during acute attacks or exacerbations. The ultrasonic data obtained before and 6 months after treatment were compared, and the therapeutic effects were evaluated.

All patients were divided into "good-effect" and "pooreffect" groups according to their treatment. In "good-effect" groups: after treatment, the symptoms of patients were significantly improved, dyspnea was relieved, hoarseness was improved. Local changes before and after treatment were compared by tracheoscopy and CT examination. Local inflammation subsided, mucosal hyperplasia was significantly improved, and the stenosis of tracheal was effectively controlled. In "poor-effect" groups: patients have no improvement in symptoms after treatment, still have dyspnea, hoarseness and other symptoms. Tracheoscopy, CT examination before and after treatment, there were still tracheostenosis $(15,16)$.

The EHFU and CEUS examination times (min) were recorded between couplant application to the end of the examination. All procedures were performed by a skilled physician with more than 3 years of ultrasound experience.

\section{Statistical analysis}

SPSS software (ver. 21.0; SPSS Inc., Chicago, IL, USA) was used for statistical analysis. Continuous data with a normal distribution are expressed as means \pm standard deviations, and categorical data as percentages. The $t$-test was used to compare continuous data among groups, and the chisquared test to compare categorical data. A $\mathrm{P}$ value $<0.05$ was taken to indicate statistical significance.

\section{Results}

\section{Characteristics of the 24 patients with CT-RP}

A total of 24 patients with confirmed CT-RP were included in this study. Ultrasound revealed 136 cartilage rings, of which 124 were abnormal. The male: female ratio was $1: 1$ (12:12) and the average age was $46 \pm 12$ (range, 26-68) years. Of all patients, $18(75 \%)$ exhibited respiratory tract involvement as the initial symptom, compared to four (17\%) with auricular collapse with respiratory tract involvement and two $(8 \%)$ with nasal collapse with respiratory tract involvement (Figure 1). Most patients (75\%) exhibited more than five damaged tracheal cartilage rings and only six (25\%) 
Page 4 of 11

Table 1 Characteristics of the 24 CT-RP patients

\begin{tabular}{|c|c|c|}
\hline Characteristics & $\mathrm{N}$ & $\%$ \\
\hline \multicolumn{3}{|l|}{ Age (y) } \\
\hline Range & $26-68$ & \\
\hline Mean \pm SD & $46 \pm 12$ & \\
\hline \multicolumn{3}{|l|}{ Sex } \\
\hline Male & 12 & 50 \\
\hline Female & 12 & 50 \\
\hline \multicolumn{3}{|l|}{ Smoke } \\
\hline Yes & 10 & 42 \\
\hline No & 14 & 58 \\
\hline \multicolumn{3}{|l|}{ First symptom } \\
\hline Respiratory symptom & 18 & 75 \\
\hline Auricular collapse & 4 & 17 \\
\hline Nasal collapse & 2 & 8 \\
\hline \multicolumn{3}{|l|}{ Tracheal cartilage rings } \\
\hline$<5$ & 6 & 25 \\
\hline$>5$ & 18 & 75 \\
\hline \multicolumn{3}{|c|}{ Respiratory interventional therapy } \\
\hline Yes & 10 & 42 \\
\hline No & 14 & 58 \\
\hline \multicolumn{3}{|l|}{ Hormone therapy } \\
\hline Yes & 19 & 79 \\
\hline No & 5 & 21 \\
\hline \multicolumn{3}{|l|}{ Therapy effect } \\
\hline Good & 18 & 75 \\
\hline Poor & 6 & 25 \\
\hline
\end{tabular}

CT-RP, cervical trachea-associated relapsing polychondritis.

had less than five damaged rings. Ten patients (42\%) received respiratory interventions and $19(79 \%)$ received hormone therapy, and while $18(75 \%)$ improved after treatment six $(25 \%)$ did not (Table 1).

\section{EHFU, CEUS, and CT manifestations in the acute stage}

In the acute phase, EHFU and CEUS revealed that the cervical tracheal cartilage rings were affected to varying degrees, exhibiting uneven cartilage walls, blurred edges, uniform or uneven cartilage thickening, uneven light spots in
Tang et al. EHFU combined with CEUS for evaluation of CT-RP

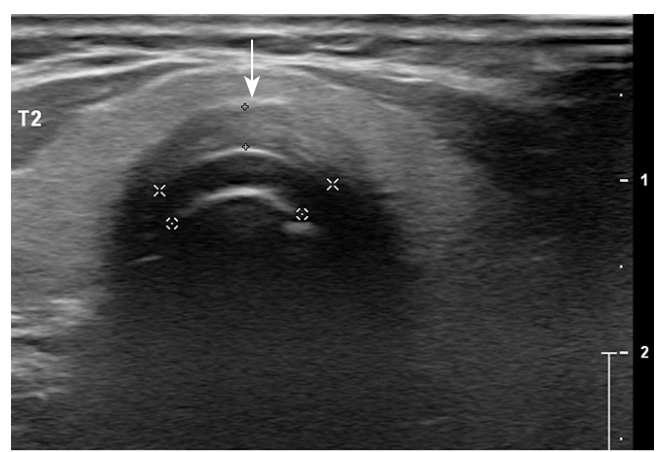

Figure 2 Cross-section EHFU image; the tracheal cartilage (arrow) exhibits non-uniform thickening, uneven echoes, and a "frosted glass" appearance. EHFU, extracorporeal high-frequency ultrasound.

cartilage and variable echoes from the spots, a "frosted glass" or "stratified" appearance, and/or calcification (Figure 2). In the echo mode without enhancement, CEUS revealed homogeneous cartilage rings. In "high echo" mode (contrast agent perfusion), CEUS revealed the surrounding soft tissue and variable thickening of the tracheal cavity mucous membrane (i.e., pathological changes in tracheal cartilage morphology; Figure 3). In six patients, the tracheal cartilage layer was deformed and thin (Figure 4). Airway CT revealed variable tracheal wall thickening and partial calcification (Figure 5).

\section{Cervical tracheal cartilage thicknesses and intratracheal diameter: comparison of ultrasound and CT measurements}

A total of 124 cervical tracheal rings exhibiting abnormal echoes were evaluated using EHFU/CEUS and CT, and the results were as follows: Thickness of the cervical trachea cartilage: EHFU, $3.657 \pm 0.52 \mathrm{~mm}, \mathrm{CT}, 3.32 \pm 0.76 \mathrm{~mm}$ $(t=1.482)$; and minimum internal transverse tracheal diameter (diameter of the narrowest segment): EHFU, $11.98 \pm 3.22 \mathrm{~mm}$, CT, $10.8 \pm 2.92 \mathrm{~mm}(t=1.005)$. The ultrasound and CT measurements did not differ significantly (all $\mathrm{P}>0.05$ ) (Table 2 and Figure 6).

\section{Evaluation of therapeutic effects}

Patients were divided into "good-effect" (75\%) and "poor-effect" (25\%) groups. Treatment effectiveness was determined based on the extent of damage to the tracheal cartilage and did not differ by age, sex, smoking status, the first symptoms, the affected tracheal cartilage rings, 

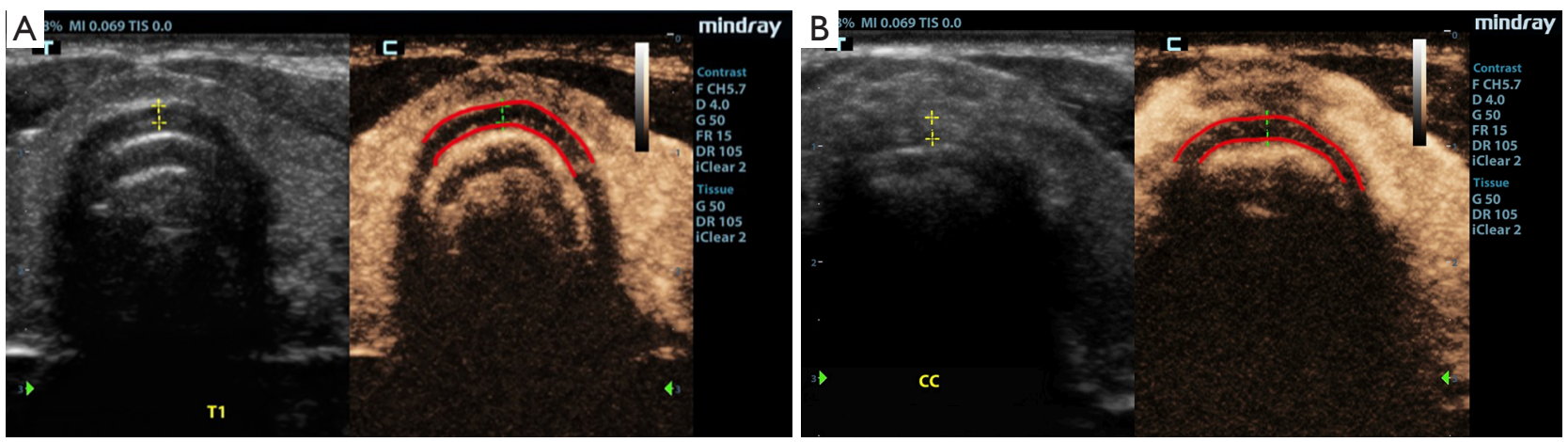

Figure 3 Cross-section CEUS image. (A,B) The tracheal cartilage layer as revealed by CEUS (red part). CEUS, contrast-enhanced ultrasound.
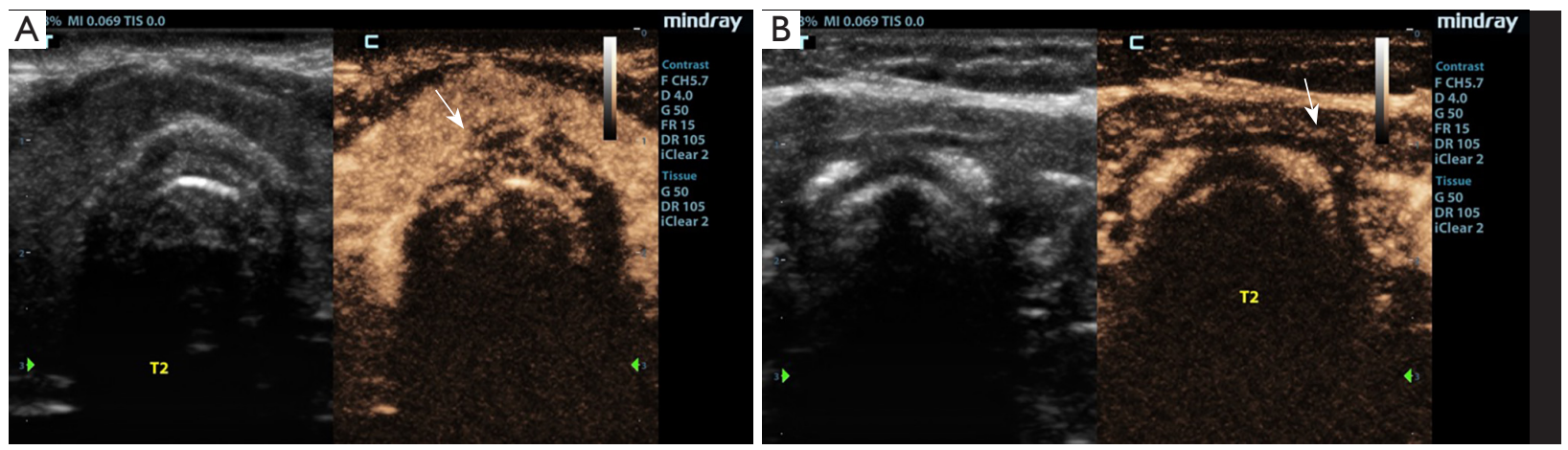

Figure 4 Cross-section CEUS image. (A,B) The tracheal cartilage layer was damaged (deformed and thinned) (arrows). CEUS, contrastenhanced ultrasound.

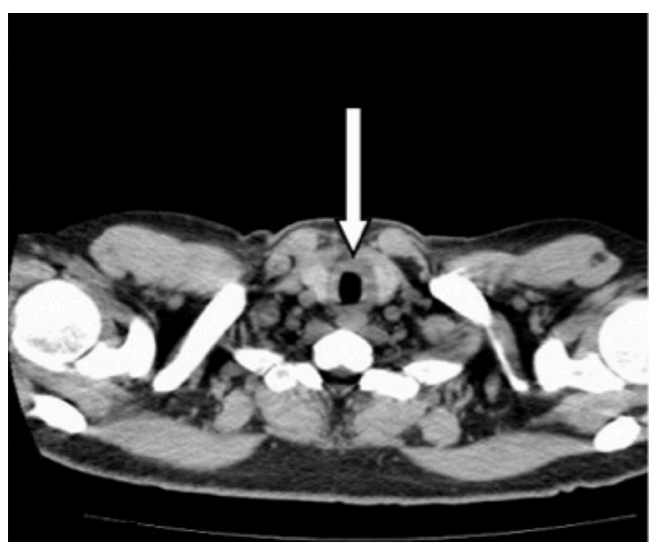

Figure 5 Longitudinal neck CT revealed that the tracheal wall was significantly thickened under the glottis (arrow). CT, computed tomography. respiratory interventional therapy, or hormonal therapy (Table 3).

In the good-effect group, the main ultrasonic manifestations were thinning of a thickened airway wall (tending toward normal), decrease or disappearance of the echo and light spots on diseased cartilage, normalization of regions with weak or no echoes, and an increase in the inner diameter of the tracheal cavity (Figure 7). These features were not seen in the poor-effect group, in which CEUS revealed damaged tracheal cartilage layers in five patients $(83 \%)$, while the good-effect group contained only one $(6 \%)$ such patient. Comparison of ultrasound measurements before and 6 months after treatment revealed that the thickness of the lesioned tracheal ring was $2.4-5 \mathrm{~mm}$ (average, $3.73 \pm 0.69 \mathrm{~mm}$ ) in the good- 
Table 2 Tracheal cartilage thickness and intratracheal diameter $(\mathrm{mm})$ of 124 cervical segments, as revealed by EHFU and CT

\begin{tabular}{lcccc}
\hline Tracheal measurement & EHFU $(\mathrm{n}=124)$ & $\mathrm{CT}(\mathrm{n}=124)$ & $t$ & $\mathrm{P}$ \\
\hline Tracheal cartilage thickness & $3.657 \pm 0.52$ & $3.32 \pm 0.76$ & 1.482 & $>0.05$ \\
Intratracheal diameter & $11.98 \pm 3.22$ & $10.8 \pm 2.92$ & 1.005 & $>0.05$ \\
\hline
\end{tabular}

EHFU, extracorporeal high-frequency ultrasound; CT, computed tomography.
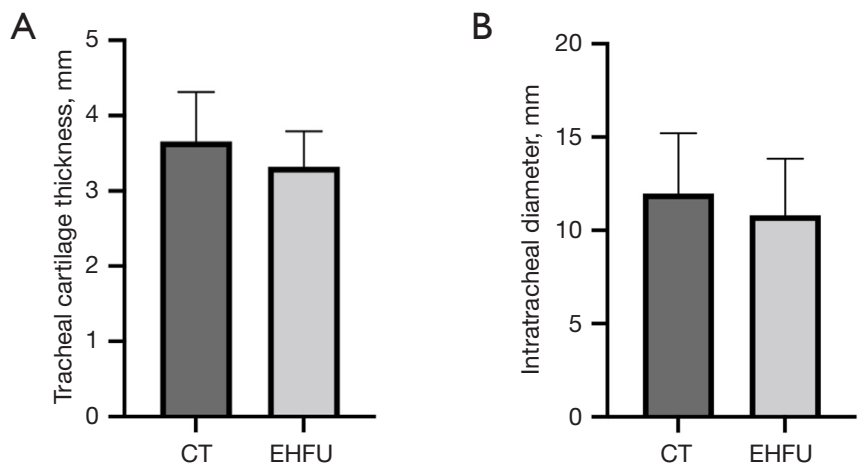

Figure 6 Comparison of ultrasound and CT measurements. Tracheal cartilage thickness (A) and intratracheal diameter (B) (mm) of 124 cervical segments, as revealed by EHFU and CT. EHFU, extracorporeal high-frequency ultrasound; CT, computed tomography.

effect group before treatment, while after treatment it was $1.7-3.9 \mathrm{~mm}$ (average, $2.98 \pm 0.62 \mathrm{~mm})(t=3.367, \mathrm{P}<0.01)$. Before treatment, the transverse diameter of the tracheal ring was $6-17 \mathrm{~mm}$ (average, $11.64 \pm 1.76 \mathrm{~mm}$ ), while after treatment it was $8-18 \mathrm{~mm}$ (average, $13.98 \pm 2.92 \mathrm{~mm}$ ) $(t=3.581, \mathrm{P}<0.01)$. In the poor-effect group, there was no significant difference in the thickness or transverse diameter of the tracheal ring before and after treatment $(3.22 \pm 0.47,12.21 \pm 3.04,3.15 \pm 0.52$ and $11.86 \pm 3.11 \mathrm{~mm}$, respectively) (Table 4 and Figure 8 ).

\section{EHFU and CEUS examination time}

The EHFU/CEUS examination time was 7-14 min (mean, $10.0 \pm 2.3 \mathrm{~min}$ ).

\section{Discussion}

$\mathrm{RP}$ is a recurrent invasive disease affecting the cartilage and connective tissues of the ear, nose, throat, trachea, eyes, joints, heart valves, blood vessels, and other organs (1) in which respiratory tracheal cartilage involvement is the most serious complication. About half of the respiratory tracheal cartilage may be involved, accounting for up to $50 \%$ of cases of mortality (17). Diagnosis is based principally on clinical manifestations (18) including recurrent ear chondritis, nonerosive polyarthritis, nasal chondritis, eye inflammation, laryngeal and/or tracheal chondritis, and cochlear and/ or vestibular damage. Michet et al. (19) proposed that RP could be diagnosed if three or more of the above criteria were met and, no histopathological confirmation was required. The diagnostic criteria are as follows: the Michet criteria are met; respiratory symptoms are evident during disease onset; there is no history of smoking or respiratory infections, chronic obstructive pulmonary disease, bronchial asthma, amyloidosis, Wegener granulomatosis, or bronchial tuberculosis; and other diseases are excluded (5). The clinical/pathological evidence, tracheal endoscopy, and chest CT data for all 24 of our patients supported a diagnosis of CT-RP.

The etiology of RP is unclear $(1,20)$, and the treatment of RP is challenging, requiring the joint efforts of multidisciplinary professionals in otorhinolaryngology, rheumatology, ophthalmology, cardiothoracic surgery and other departments. The therapy usually involves combinations of cortisol-reducing drugs, non-steroidal antiinflammatory drugs, and other immunosuppressive drugs, as these reduce the severity and number of relapses (21). Patients with CT-RP should be treated aggressively to improve the patient's quality of life and alleviate symptoms. 
Table 3 Univariate analysis of treatment effects

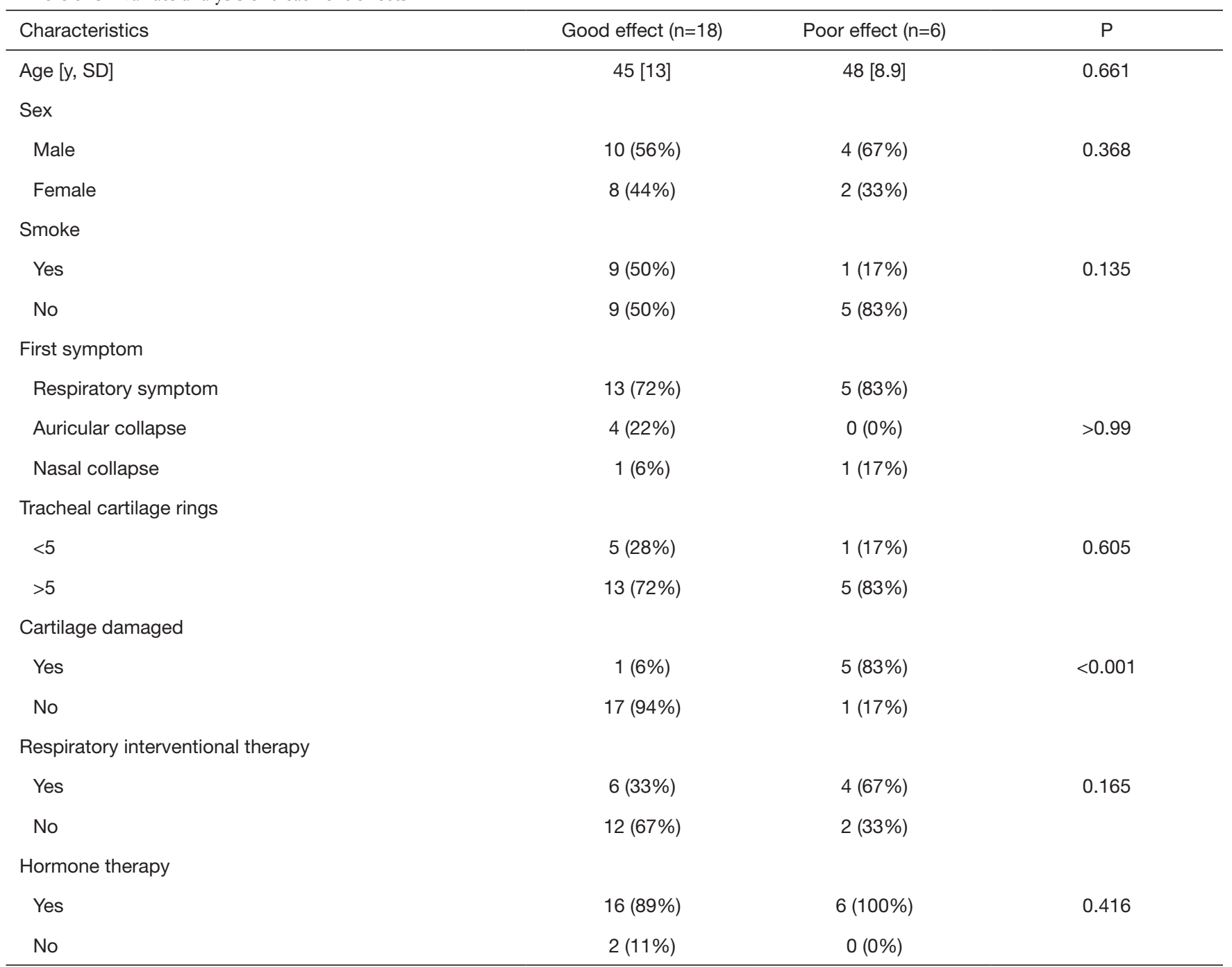

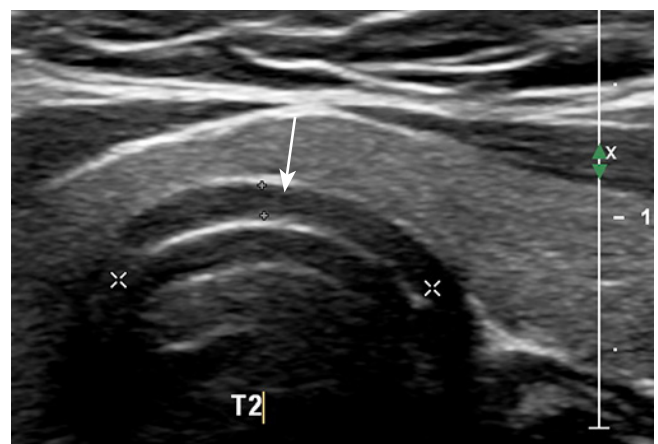

Figure 7 Ultrasound image of tracheal cartilage obtained 6 months after treatment. The cross-section (arrow) reveals a regular morphology, clear boundaries, smooth edges, and uniform echogenicity.
Glucocorticoids are the most commonly used drugs and the basis of treatment, combined with immunosuppressants for patients with severe respiratory tract involvement $(22,23)$. For patients with laryngeal and tracheal stenosis, balloon dilation under bronchoscopy, stent placement in the airway, or tracheotomy can relieve dyspnea, improve ventilation and expectoration, and use ventilator to assist ventilation when necessary (23). Airway management is extremely challenging, and treatment must be personalized (4). Timely monitoring of cartilage lesion progression aids treatment planning and may prolong survival.

Currently, airway CT and tracheoscopy are the most used auxiliary methods for CT-RP diagnosis and evaluation. However, as mentioned in the Introduction section, both 
Table 4 Tracheal cartilage thickness and intratracheal diameter $(\mathrm{mm})$ before and after treatment, as revealed by EHFU

\begin{tabular}{|c|c|c|c|c|}
\hline Group & Before treatment & After treatment & $t$ & $\mathrm{P}$ \\
\hline \multicolumn{5}{|c|}{ Tracheal cartilage thickness } \\
\hline Good effect $(n=98)$ & $3.73 \pm 0.69$ & $2.98 \pm 0.62$ & 3.367 & $<0.01$ \\
\hline Poor effect $(n=26)$ & $3.22 \pm 0.47$ & $3.15 \pm 0.52$ & 0.348 & $>0.05$ \\
\hline \multicolumn{5}{|l|}{ Intratracheal diameter } \\
\hline Poor effect $(n=26)$ & $12.21 \pm 3.04$ & $11.86 \pm 3.11$ & 0.338 & $>0.05$ \\
\hline
\end{tabular}

EHFU, extracorporeal high-frequency ultrasound.

A

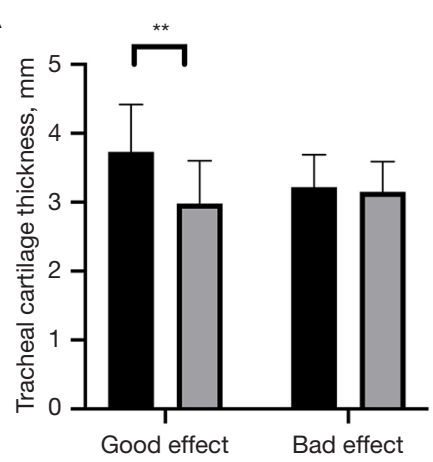

B

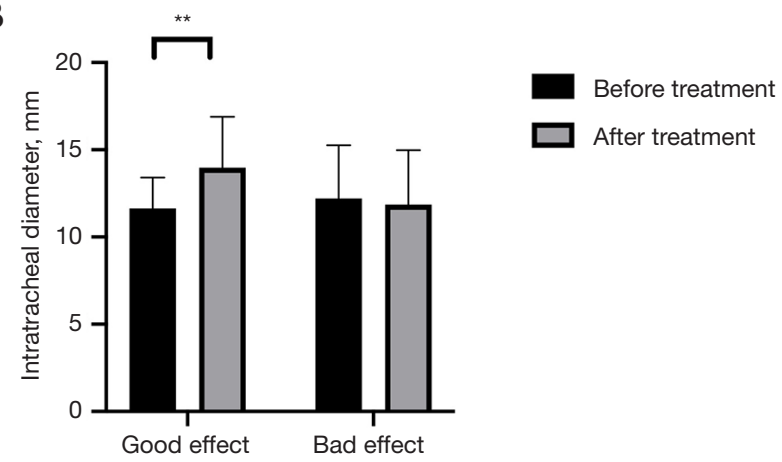

Figure 8 Evaluation of therapeutic effects. Tracheal cartilage thickness (A) and intratracheal diameter (B) (mm) before and after treatment, as revealed by EHFU. **, $\mathrm{P}<0.01$. EHFU, extracorporeal high-frequency ultrasound.

have limitations. It has been reported that fluorine-18 fludeoxyglucose positron emission tomography-CT $\{[18]$ F-FDG PET-CT\} can be used for the diagnosis of CT$\mathrm{RP}$, and the hyperphotography of the inflammatory foci is usually shown to be [18]F-FDG, which is helpful for the diagnosis of early CT-RP (24-26). The [18]F-FDG PET-CT manifestations of CT-RP have certain specificity and can assess the early respiratory involvement of RP. Moreover, by localizing the sites of active inflammation, [18]F-FDG PET-CT may guide the selection of a biopsy site (27). However, [18]F-FDG PET-CT has poor display rate for finer structures, and this examination has radiation and is expensive. EHFU is a non-invasive, non-radiative, convenient real-time method for revealing the structure of the anterior wall of the cervical trachea and surrounding soft tissue $(13,28)$. The newest ultrasound probes operate at higher frequencies and provide enhanced imaging quality, clearly revealing the cartilage morphology of the anterior and neck tracheal wall $(11,12)$. In a cadaver study, Tsui et al. (10) found that transverse EHFU sections showed the annular tracheal rings of the anterior wall of the trachea, the numbers of which varied by the length of the neck, and the surrounding soft tissue, while longitudinal sections revealed a "bead-like" arrangement of the rings and surrounding soft tissue. The EHFU, CT, and autopsy data were in good agreement. EHFU can be used to assess the extent of subglottic stenosis, and Lakhal et al. and Salgo et al. $(29,30)$ reported their EHFU data were in excellent agreement with magnetic resonance imaging and bronchoscopic measurements of tracheal transverse diameters at the cricoid level. In a study enrolling six patients with tracheal stenosis and 20 normal controls, Shih et al. (31) evaluated the feasibility of EHFU for tracheal lesion evaluation and for diagnosis and follow-up of patients with tracheal stenoses of various etiologies, and the findings were similar to those of our previous study.

Normal tracheal cartilage is weakly echoic (nearly echoless). Morphologically, apart from the circular cartilage (which is a closed "ring"), the tracheal cartilage "rings" are in fact notch-shaped, appearing as "Cs" (11). In ultrasound 
cross-sections, normal cervical tracheal cartilages present as smooth, arc-shaped, thin, and weakly echoic bands with uniform internal echoes. The EHFU and CEUS characteristics of the 24 CT-RP patients in this study were as follows: almost all detectable tracheal cartilages were involved, exhibiting uniform or non-uniform thickening; the echoes at the junctions of the tracheal walls were weakened, and the edges were blurred; the echoes of the tracheal cartilage increased to varying extents, exhibiting a "frosted glass" pattern and sometimes appearing as layered or calcified; and the lumen shrank concentrically. These findings were consistent with the report by Rafeq et al. (5), in which specific CT manifestations included subglottic stenosis, tracheal stenosis, tracheal wall thickening, calcification, and tracheal softening. We found that the EHFU/CEUS and CT measurements of the cervical tracheal cartilage thickness and minimum internal transverse diameter were essentially identical.

This study is the first to use CEUS to analyze tracheal wall lesions in CT-RP. Microcirculation perfusion imaging of the vascular pool clearly revealed the distribution and perfusion of tissue microvessels $(14,32)$. Tracheal cartilage, like all cartilage, lacks blood, lymphatic vessels, and nerves, resulting in ultrasound microbubble contrast agent being unable to enter it. Therefore, in CEUS mode, the nonenhanced tracheal cartilage layer is anechoic and dark and clearly distinguishable from surrounding tissues. CEUS can also be used to evaluate other tracheal diseases. On conventional EHFU, some of our patients showed echoes from cartilage rings as the gray level increased, and reduced echoes for the surrounding soft tissues (such as the connective tissue layer) caused by inflammatory changes, making it difficult to clearly distinguish the tracheal cartilage. However, after the addition of CEUS, all tracheal cartilages were distinguished, allowing more accurate assessment of illness, and improving the data, which is especially important when tracheal injuries are serious.

The tracheal cartilage layer was damaged in six of our patients, and CEUS clearly revealed cartilage destruction, deformation, and thinning. Chang et al. (33) suggested that recurrent attacks were associated with cartilage destruction, and that cartilage damage and scar hyperplasia could trigger localized or global airway softening, collapse, and stenosis. After 6 months of follow-up, five of the patients with cartilage injuries had responded poorly to treatment, and despite large doses of hormones and methotrexate, symptoms of tracheal stenosis persisted, and even bronchial stenting and tracheotomy were ineffective. Therefore, early and accurate detection and assessment of tracheal cartilage lesions in patients with CT-RP is of great practical significance, and prompt treatment may delay cartilage destruction and relieve the disease.

In summary, our preliminary results indicate that EHFU combined with CEUS clearly reveals cervical tracheal cartilage, tracheal walls, the intratracheal CT-RP diameter, and microstructural changes in major cartilage supports. As ultrasound detects tracheal cartilage thickening and relief and echo changes after treatment, it is reliable in evaluating treatment effects and dynamically monitoring changes in disease status. Ultrasound provides timely and detailed information on tracheal cartilage lesions. However, EHFU combined with CEUS also has certain limitations. It can only display the trachea in the cervical segment. Due to the block of the sternum on the surface of the body, the trachea below the sternum cannot be examined by external ultrasound, which needs to be combined with other examination methods. In addition, as we retrospectively analyzed only a small number of cases, larger prospective studies are required. Ultrasound may become routine for early CT-RP diagnosis, monitoring of treatment efficacy, and follow-up.

\section{Acknowledgments}

Funding: None.

\section{Footnote}

Reporting Checklist: The authors have completed the STROBE reporting checklist. Available at https://dx.doi. org/10.21037/atm-21-6175

Data Sharing Statement: Available at https://dx.doi. org/10.21037/atm-21-6175

Conflicts of Interest: All authors have completed the ICMJE uniform disclosure form (available at https://dx.doi. org/10.21037/atm-21-6175). The authors have no conflicts of interest to declare.

Ethical Statement: The authors are accountable for all aspects of the work in ensuring that questions related to the accuracy or integrity of any part of the work are appropriately investigated and resolved. All procedures performed in this study involving human participants were in accordance with the Declaration of Helsinki (as revised in 


\section{Page 10 of 11}

2013). This study was approved by the Scientific Research Ethics Review Committee of The First Affiliated Hospital of Guangzhou Medical University [No. (2021)140], and all subjects gave written informed consent.

Open Access Statement: This is an Open Access article distributed in accordance with the Creative Commons Attribution-NonCommercial-NoDerivs 4.0 International License (CC BY-NC-ND 4.0), which permits the noncommercial replication and distribution of the article with the strict proviso that no changes or edits are made and the original work is properly cited (including links to both the formal publication through the relevant DOI and the license). See: https://creativecommons.org/licenses/by-nc-nd/4.0/.

\section{References}

1. Trentham DE, Le CH. Relapsing polychondritis. Ann Intern Med 1998;129:114-22.

2. de Montmollin N, Dusser D, Lorut C, et al. Tracheobronchial involvement of relapsing polychondritis. Autoimmun Rev 2019;18:102353.

3. Belot A, Duquesne A, Job-Deslandre C, et al. Pediatriconset relapsing polychondritis: case series and systematic review. J Pediatr 2010;156:484-9.

4. Ernst A, Rafeq S, Boiselle P, et al. Relapsing polychondritis and airway involvement. Chest 2009;135:1024-30.

5. Rafeq S, Trentham D, Ernst A. Pulmonary manifestations of relapsing polychondritis. Clin Chest Med 2010;31:513-8.

6. Suyama Y, Ishimoto SI, Hagiwara K. Clinical Images: Arytenoid Chondritis. Arthritis Rheumatol 2017;69:1193.

7. Lin ZQ, Xu JR, Chen JJ, et al. Pulmonary CT findings in relapsing polychondritis. Acta Radiol 2010;51:522-6.

8. Lee KS, Ernst A, Trentham DE, et al. Relapsing polychondritis: prevalence of expiratory CT airway abnormalities. Radiology 2006;240:565-73.

9. Miyazu Y, Miyazawa T, Kurimoto N, et al. Endobronchial ultrasonography in the diagnosis and treatment of relapsing polychondritis with tracheobronchial malacia. Chest 2003;124:2393-5.

10. Tsui B, Ip V, Walji A. Airway sonography in live models and cadavers. J Ultrasound Med 2013;32:1049-58.

11. Diwakar A, Adam RJ, Michalski AS, et al. Sonographic evidence of abnormal tracheal cartilage ring structure in cystic fibrosis. Laryngoscope 2015;125:2398-404.

12. Prasad A, Yu E, Wong DT, et al. Comparison of sonography and computed tomography as imaging tools for assessment of airway structures. J Ultrasound Med

\section{Tang et al. EHFU combined with CEUS for evaluation of CT-RP}

2011;30:965-72.

13. Singh M, Chin KJ, Chan VW, et al. Use of sonography for airway assessment: an observational study. J Ultrasound Med 2010;29:79-85.

14. Cantisani V, Di Leo N, David E, et al. Role of CEUS in Vascular Pathology. Ultraschall Med 2021;42:348-66.

15. Rose E, Ferrada MA, Quinn KA, et al. Physician Global Assessment as a Disease Activity Measure for Relapsing Polychondritis. Arthritis Care Res (Hoboken) 2021. doi: 10.1002/acr.24574.

16. Rose E, Ferrada MA, Quinn KA, et al. Discordance in Patient and Physician Global Assessment in Relapsing Polychondritis. Rheumatology (Oxford) 2021. doi: 10.1093/rheumatology/keab587.

17. Kent PD, Michet CJ Jr, Luthra HS. Relapsing polychondritis. Curr Opin Rheumatol 2004;16:56-61.

18. Dion J, Costedoat-Chalumeau N, Sène D, et al. Relapsing Polychondritis Can Be Characterized by Three Different Clinical Phenotypes: Analysis of a Recent Series of 142 Patients. Arthritis Rheumatol 2016;68:2992-3001.

19. Michet CJ Jr, McKenna CH, Luthra HS, et al. Relapsing polychondritis. Survival and predictive role of early disease manifestations. Ann Intern Med 1986;104:74-8.

20. Puéchal X, Terrier B, Mouthon L, et al. Relapsing polychondritis. Joint Bone Spine 2014;81:118-24.

21. Tillie-Leblond I, Wallaert B, Leblond D, et al. Respiratory involvement in relapsing polychondritis. Clinical, functional, endoscopic, and radiographic evaluations. Medicine (Baltimore) 1998;77:168-76.

22. Kingdon J, Roscamp J, Sangle S, et al. Relapsing polychondritis: a clinical review for rheumatologists. Rheumatology (Oxford) 2018;57:1525-32.

23. Hazra N, Dregan A, Charlton J, et al. Incidence and mortality of relapsing polychondritis in the UK: a population-based cohort study. Rheumatology (Oxford) 2015;54:2181-7.

24. Lei W, Zeng H, Zeng DX, et al. (18)F-FDG PET-CT: a powerful tool for the diagnosis and treatment of relapsing polychondritis. Br J Radiol 2016;89:20150695.

25. Baudart $P$, Aouba A, Beaufrère $M$, et al. FDG PET-CT as a powerful tool for diagnosing and monitoring treatment outcomes of relapsing polychondritis. Eur J Nucl Med Mol Imaging 2018;45:669-70.

26. Sharma A, Kumar R, Mb A, et al. Fluorodeoxyglucose positron emission tomography/computed tomography in the diagnosis, assessment of disease activity and therapeutic response in relapsing polychondritis. Rheumatology (Oxford) 2020;59:99-106. 
27. Sato M, Hiyama T, Abe T, et al. F-18 FDG PET/CT in relapsing polychondritis. Ann Nucl Med 2010;24:687-90.

28. Eom K, Moon K, Seong Y, et al. Ultrasonographic evaluation of tracheal collapse in dogs. J Vet Sci 2008;9:401-5.

29. Lakhal K, Delplace X, Cottier JP, et al. The feasibility of ultrasound to assess subglottic diameter. Anesth Analg 2007;104:611-4.

30. Salgo B, Schmitz A, Henze G, et al. Evaluation of a new recommendation for improved cuffed tracheal tube size selection in infants and small children. Acta Anaesthesiol
Scand 2006;50:557-61.

31. Shih JY, Lee LN, Wu HD, et al. Sonographic imaging of the trachea. J Ultrasound Med 1997;16:783-90.

32. Desailly Y, Tissier AM, Correas JM, et al. Contrast enhanced ultrasound by real-time spatiotemporal filtering of ultrafast images. Phys Med Biol 2017;62:31-42.

33. Chang SJ, Lu CC, Chung YM, et al. Laryngotracheal involvement as the initial manifestation of relapsing polychondritis. J Chin Med Assoc 2005;68:279-82.

(English Language Editor: B. Draper)

Cite this article as: Tang J, He W, Zhang Y, Tang Q, He N, Liang Z, Li S. Extracorporeal high-frequency combined with contrast-enhanced ultrasound: a novel imaging method for detection and treatment evaluation of patients with cervical trachea-associated relapsing polychondritis. Ann Transl Med 2021;9(24):1785. doi: 10.21037/atm-21-6175 